\title{
Effect of optic disc size on correlation between Bruch's membrane opening-minimum rim width and peripapillary retinal nerve fibre layer thickness
}

\author{
Hyun-kyung Cho $\mathbb{D}^{1,2} \cdot$ Jong Moon Park ${ }^{1,2} \cdot$ Changwon $\mathrm{Kee}^{3}$
}

Received: 20 September 2018 / Revised: 26 May 2019 / Accepted: 24 June 2019 / Published online: 8 July 2019

(c) The Author(s), under exclusive licence to The Royal College of Ophthalmologists 2019

\begin{abstract}
Objectives To investigate the effect of optic disc size on correlation between Bruch's membrane opening-minimum rim width (BMO-MRW) and peripapillary retinal nerve fibre layer (RNFL) thickness from three scan circles.

Methods In this retrospective, observational study, non-glaucomatous eyes without visible RNFL defect or visual field loss were included. A total of 101 subjects were distributed into three groups based on disc size: group $1(n=26)$, small disc (disc area $\left.<1.63 \mathrm{~mm}^{2}\right)$; group $2(n=40)$, regular size disc (disc area: $\left.1.63 \sim 2.43 \mathrm{~mm}^{2}\right)$; and group $3(n=35)$, large disc (disc area $>2.43 \mathrm{~mm}^{2}$ ). All patients underwent standard ophthalmic examinations including confocal scanning laser tomography and spectral-domain optical coherence tomography.

Results Global BMO-MRW was the thickest in group $1\left(314.96 \pm 60.38 \mu \mathrm{m}, \mathrm{BMO}\right.$ area: $\left.1.72 \pm 0.45 \mathrm{~mm}^{2}\right)$, followed by that in group $2\left(259.03 \pm 40.04 \mu \mathrm{m}, \mathrm{BMO}\right.$ area: $\left.2.29 \pm 0.31 \mathrm{~mm}^{2}\right)$. It was the thinnest in group $3(236.74 \pm 31.21 \mu \mathrm{m}, \mathrm{BMO}$ area: $2.91 \pm 0.31 \mathrm{~mm}^{2} ; p<0.001$, Kruskal-Wallis test). Correlation between global BMO-MRW value and RNFL thickness was the strongest in group 3 (Spearman's rho $=0.656)$, followed by that in group $2($ rho $=0.572)$. It was the weakest in group 1 $($ rho $=0.147)$. There was no significant difference in global RNFL thickness by disc size from either the $3.5 \mathrm{~mm}, 4.1 \mathrm{~mm}$, or $4.7 \mathrm{~mm}$ diameter scan circles (all $p>0.05$ ).

Conclusions Correlation between BMO-MRW values and RNFL thickness differed significantly according to disc size. Thus, when we assess BMO-MRW in relation to RNFL thickness, disc size may need to be taken into consideration.
\end{abstract}

\section{Introduction}

Glaucoma involves the injury of retinal ganglion cells (RGC) and their axons, causing defect of the retinal nerve fibre layer (RNFL) and the neuroretinal rim and resulting in visual field defect [1]. Detecting early structural defects is more important than functional loss in early glaucoma

Hyun-kyung Cho

chohk@gnu.ac.kr

kanojo99@hanmail.net

1 Department of Ophthalmology, Gyeongsang National University Changwon Hospital, Gyeongsang National University, School of Medicine, Changwon, Republic of Korea

2 Institute of Health Sciences, School of Medicine, Gyeongsang National University, Jinju, Republic of Korea

3 Department of Ophthalmology, Samsung Medical Center, Sungkyunkwan University School of Medicine, Seoul, Republic of Korea diagnosis [2, 3] because detectable structural abnormality may precede functional abnormality at individual level [4-6]. In early stage of glaucoma or in glaucoma suspect, structural change is minimal. Therefore, results from different structural tests may not correspond with each other. For example, Bruch's membrane opening-minimum rim width (BMO-MRW) and peripapillary RNFL thickness from optical coherence tomography (OCT) may not correlate with each other. If one structural test says normal, whereas the other suggests abnormal, it may yield confusion to clinicians in the diagnosis of early glaucoma. This early stage of glaucoma is particularly important because decision for the initiation of lifetime treatment is required.

Recently, BMO-MRW as a new parameter has been introduced in the evaluation of optic disc [7-11]. It is the minimum length measured from the BMO to the internal limiting membrane (Fig. 1). BMO-MRW provides not only consistent borders, but also a geometrically more accurate assessment of the neuroretinal rim than conventional ophthalmic examination [7-9, 12]. Recent studies have 
(a)

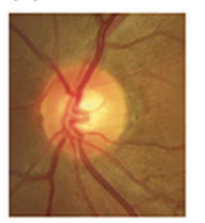

(b)

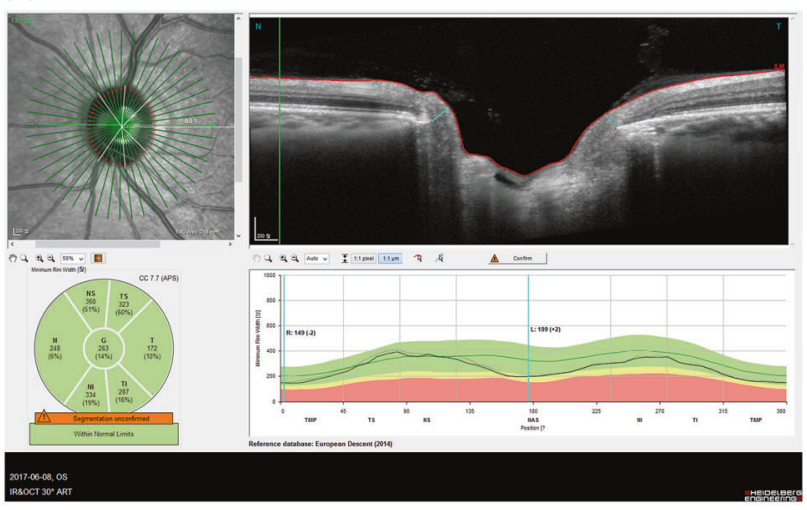

(d)

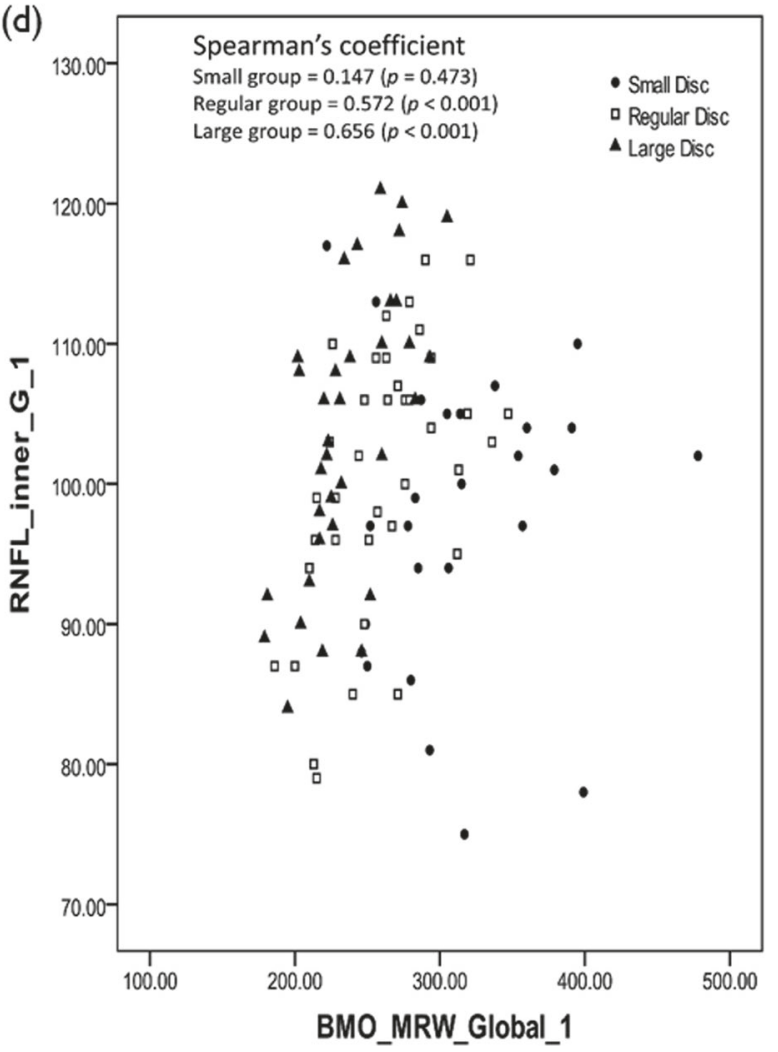

demonstrated that BMO-MRW gives better diagnostic performance for glaucoma than conventional neuroretinal rim parameters [13-15].

Diagnosing glaucoma in very large or very small optic discs is challenging for clinicians. In large optic discs, the neuroretinal rim on funduscopy appears thinned and the optic disc cup appears large, whereas the number of RGC (c)

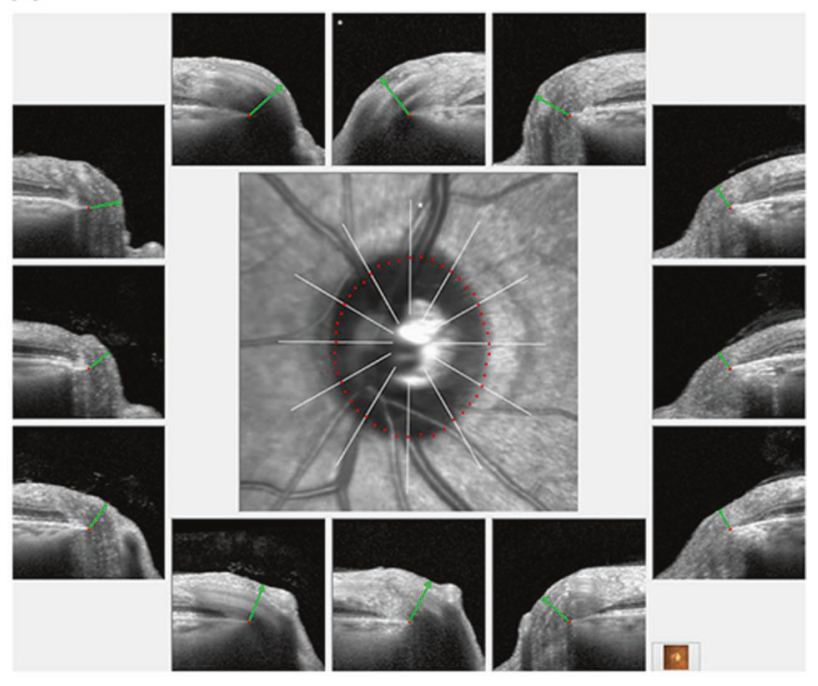

axons is the same as or higher than that in regular size discs. Therefore, patients with large optic disc are often classified as glaucoma suspects owing to large cupping. On the contrary, in small optic discs, the neuroretinal rim on fundus photography appears thicker. It frequently has no or just a small cupping. If optic disc size influences the correlation between BMO-MRW and RNFL thickness and suggests 
Fig. 1 Bruch's membrane opening-minimum rim width (BMO-MRW) and BMO overview. b BMO-MRW is a new parameter in the assessment of optic disc. It is the minimum length measured from BMO (red dot) to the internal limiting membrane (red line). This figure shows global BMO-MRWs and BMO-MRWs for six Garway-Heath sectors. Sectoral analysis was performed according to the individual eyespecific axis, the FoBMO axis. This is the axis between the fovea and the BMO centre. It can lead to more-accurate sectoral analysis taking cyclotorsion of individual eyes into consideration. c BMO overview shows BMO margin of the disc and detailed BMO-MRW measurement cuts from 12 directions. a Disc photography of the same optic disc for reference. $\mathbf{d}$ Correlation between Bruch's membrane opening-minimum rim width and retinal nerve fibre layer thickness according to optic disc size. Scatter plot showing correlation between global Bruch's membrane opening-minimum rim width (BMO-MRW) and global peripapillary retinal nerve fibre layer (RNFL) thickness according to optic disc size from inner scan circle. Small disc group showed no significant correlation between global BMO-MRW and RNFL thickness (Spearman's rho $=0.147, p=0.473$ ). BMO-MRWs for all six Garway-Heath sectors showed no significant correlation with RNFL thickness either. Regular size disc group showed significant correlation between global BMO-MRW and RNFL thickness (Spearman's rho $=0.572, p<$ 0.001 ), although regional sectors showed variable correlations. Large disc group showed significant correlation between global BMO-MRW and RNFL thickness (Spearman's rho $=0.656, p<0.001$ ). BMOMRWs of all six sectors also showed significant correlations with RNFL thickness. *Values are Spearman's correlation coefficient rho ( $p$ value). ${ }^{*} p$ values $<0.017$ were considered to be statistically significant owing to multiple comparison

different display of normal or abnormal, this factor should be considered.

Spectralis spectral-domain OCT (Glaucoma Module Premium Edition, Heidelberg Engineering, Germany) provides three circle $\mathrm{B}$-scans (3.5, 4.1 and $4.7 \mathrm{~mm}$ diameter) for RNFL thickness along with 24 radial B-scans for BMOMRW (Fig. 1b, c). Therefore, structural test results of four maps are provided to clinicians. If these four test results suggest differently, they can cause confusion to diagnose glaucoma, especially in the early stage. The most commonly used scan circle for peripapillary RNFL thickness is the inner circle partly because inner RNFL thickness shows the largest global and sectoral areas under the receiver operating characteristic curves among three scan circle sizes [16], whereas other OCT software does not provide middle or outer scan circle sizes. However, the impact of scan circle size on different disc sizes or its correlation with BMOMRW has not been studied yet. Normative database of RNFL decreases as the scan circle goes from inner to middle and outer circle and when diameter increases. If RNFL thickness from three scan circle sizes suggests different findings according to optic disc size, it should also be considered in the diagnosis of glaucoma.

We aimed to see if optic disc size might affect the correlation between BMO-MRW and RNFL thickness from three scan circle sizes. We investigated BMO-MRW and RNFL thickness from three scan circles according to optic disc size and correlations between them by disc size (small, regular and large). Moreover, we intended to see if there was a difference in peripapillary RNFL thickness by optic disc size from each scan circle of inner, middle and outer circles.

\section{Methods}

This retrospective observational, cross-sectional study was performed according to the tenets of the Declaration of Helsinki. It was approved by the Institutional Review Board of Gyeongsang National University Changwon Hospital, Gyeongsang National University School of Medicine. The requirement of informed consent was exempted owing to its retrospective nature.

\section{Subjects}

Among 173 patients with non-glaucomatous condition without other optic nerve or retinal disorders who were evaluated in glaucoma clinic at Gyeongsang National University Changwon Hospital and performed VF test, 101 subjects (101 eyes) were included in this study in accordance with the criteria described below. Subjects without visible RNFL defect, with reliable standard automated perimetry results of within normal limits (Humphrey Field Analyzer 30-2) and with baseline intraocular pressure (IOP) $<21 \mathrm{mmHg}$ were included. We included relatively normal subjects and non-glaucomatous eyes to represent the normal group in clinical setting. The purpose of these inclusion criteria was to provide practical information for clinicians in the assessment of glaucoma. These glaucoma evaluations were performed by a single examiner (H-K C).

We randomly selected only one eye for inclusion. We excluded fellow eyes in unilateral glaucoma because of their potential effects on RNFL or BMO-MRW that might be thinner than in normal controls. We excluded those with high myopia or hyperopia of $>6.0$ dioptres and those with history of any ocular surgery including refractive surgery (LASIK or LASEK) or optic neuropathy other than glaucoma. All subjects underwent standard ophthalmic examinations.

We classified subjects into three groups depending on disc area obtained by confocal scanning laser tomography (CSLT). Subjects with disc area of 1.63-2.43 $\mathrm{mm}^{2}$ (based on software normative data range) were assigned to the regular disc group (group 2). Those with disc area of $<1.63$ $\mathrm{mm}^{2}$ were placed in the small disc group (group 1), whereas those with disc area of $>2.43 \mathrm{~mm}^{2}$ were allocated to the large disc group (group 3).

\section{OCT}

OCT imaging of spectral-domain was performed using Glaucoma Module Premium Edition. Twenty-four radial 
B-scans were obtained for BMO-MRW. For peripapillary RNFL thickness, three scan circles $(3.5,4.1$ and $4.7 \mathrm{~mm}$ in diameter for inner, middle and outer, respectively) were obtained. Well-centred scans with correct retinal segmentation and quality score $>20$ were accepted. Data acquisition and analyses of OCT were performed according to individual eye-specific axis (FoBMO axis). This is the axis between the BMO centre and fovea. It can lead to more accurate sectoral analysis considering cyclotorsion of individual eyes and more accurate comparison with normative data than conventional method [7]. BMO area was also obtained for comparison with disc area by CSLT.

\section{CSLT, perimetry}

An experienced technician performed CSLT using HRT3 software (Heidelberg Engineering, Germany). Exclusion criteria were: mean pixel height standard deviation $>30 \mathrm{~mm}$, decentration of images, underillumination and moving artefacts for image quality. We used a Humphrey Field Analyzer (HFA model 840; Humphrey Instruments Inc, San Leandro, CA, USA) for perimetry with central 30-2 programme of Swedish Interactive Threshold Algorithm standard strategy.

\section{Statistical analysis}

Continuous values of the three groups were initially tested for normality and homoscedasticity using Shapiro-Wilk test and Leven's $F$ test, respectively. Differences of continuous values in these three groups were evaluated by Kruskal-Wallis test because these values did not satisfy the assumption of normality.

We compared baseline characteristics or disc parameters among the three groups using Kruskal-Wallis test. For disc parameters or RNFL thickness between groups, we used Kruskal-Wallis test with Donn's post hoc test. Statistical significance was considered when $p$ value was $<0.05$.

We evaluated correlations between BMO-MRW and RNFL thickness with Spearman's correlation coefficient rho. Statistical significance was considered when $p$ was $<0.017$ owing to multiple comparisons. All statistical analyses were performed using SPSS software version 24.0 (IBM Corp., Chicago, IL, USA).

\section{Results}

\section{Baseline characteristics}

We included a total of 101 subjects for the final analysis: 26 subjects in the small disc group (group 1), 40 in the regular disc group (group 2) and 35 in the large disc group (group 3). The mean age of all included subjects was 45.31 \pm 13.66 years. Of these subjects, $55.4 \%$ were men $(56 / 101)$ and $44.5 \%$ were women $(45 / 101)$. The mean spherical equivalent was $-1.31 \pm 2.32$. Baseline IOP was $15.05 \pm$ $2.52 \mathrm{mmHg}$. There were no significant differences in baseline characteristics including family history of glaucoma among the three groups (all $p>0.05$ ) (Table 1). The presence of $\beta$-peripapillary atrophy showed no significant difference either among the three groups $(p=0.751)$ (Table 1).

The mean disc area obtained with CSLT (HRT3) was $1.41 \pm 0.21 \mathrm{~mm}^{2}$ in group $1,2.08 \pm 0.21 \mathrm{~mm}^{2}$ in group 2 and $2.75 \pm 0.24 \mathrm{~mm}^{2}$ in group 3. Significant differences in disc area and other disc parameters were also noted among the three groups (all $p<0.0001$, Kruskal-Wallis test). However, there were no significant differences in rim volume among the three groups $(p=0.676)$ (Table 1$)$.

\section{Bruch's membrane opening parameters in each group}

Mean BMO area was $1.72 \pm 0.45 \mathrm{~mm}^{2}$ in group $1,2.29 \pm$ $0.31 \mathrm{~mm}^{2}$ in group 2 and $2.91 \pm 0.31 \mathrm{~mm}^{2}$ in group 3 . There were significant differences in BMO area among the three groups $(p<0.0001)$. Disc area obtained by HRT3 and BMO area obtained by OCT also showed strong correlations (Spearman's rho $=0.852, p<0.0001)$. The mean global BMO-MRW was $314.96 \pm 60.38 \mu \mathrm{m}$ in group $1,259.03 \pm$ $40.04 \mu \mathrm{m}$ in group 2 and $236.74 \pm 31.21 \mu \mathrm{m}$ in group 3 , showing statistically significant differences among the three groups $(p<0.0001)$. Specifically, global BMO-MRW was significantly thicker in group 1 than that in group 2 . It was also significantly thicker in group 2 than that in group 3 (both $p<0.05$, Kruskal-Wallis test with Donn's post hoc test). BMO-MRW from all six Garway-Heath sectors showed significant differences among the three groups (all $p<0.05$ ). The mean FoBMO angle was $-6.63 \pm 3.36^{\circ}$, showing no significant differences among the three groups $(p=0.741)$ (Table 2).

\section{RNFL thickness from three scan circles in each group}

The mean global RNFL thicknesses from the inner circle was $97.65 \pm 10.53 \mu \mathrm{m}$ in group $1,100.13 \pm 9.62 \mu \mathrm{m}$ in group 2 and $103.77 \pm 10.26 \mu \mathrm{m}$ in group 3, showing no significant differences among the three groups (all $p>0.05$ ). There were no significant differences in RNFL thickness from other sectors among the three groups (all $p>0.05$ ) except for the nasal sector $(p<0.001)$ (Table 3$)$. The mean global RNFL thickness from the middle scan circle or the outer scan circle showed no significant difference among the three groups (all $p>0.05$ ). Sectoral RNFL thickness from middle and outer scan circles demonstrated the same tendency as results from the inner scan circle (Table 3). 
Table 1 Baseline characteristics and optic disc characteristics of each group by disc size

\begin{tabular}{|c|c|c|c|c|}
\hline Characteristics & $\begin{array}{l}\text { Group 1: small disc } \\
(n=26)\end{array}$ & $\begin{array}{l}\text { Group 2: regular disc } \\
(n=40)\end{array}$ & $\begin{array}{l}\text { Group 3: large disc } \\
(n=35)\end{array}$ & $p$ values \\
\hline Age (year) & $44.27 \pm 15.34$ & $46.33( \pm 13.78)$ & $44.91( \pm 12.5)$ & 0.731 \\
\hline Female gender $(\%)$ & $11(42.3 \%)$ & $19(47.5 \%)$ & $15(42.8 \%)$ & 0.904 \\
\hline $\begin{array}{l}\text { Family history of } \\
\text { glaucoma }(\%)\end{array}$ & $2(7.7 \%)$ & $1(2.5 \%)$ & $2(5.7 \%)$ & 0.622 \\
\hline Spherical equivalent (D) & $-1.47 \pm 2.94$ & $-1.39( \pm 2.2)$ & $-1.1( \pm 1.97)$ & 0.504 \\
\hline $\mathrm{CCT}(\mu \mathrm{m})$ & $547.79 \pm 51.87$ & $548.08( \pm 32.95)$ & $548.67( \pm 40.33)$ & 0.960 \\
\hline Baseline IOP (mmHg) & $14.88 \pm 3.19$ & $15.03( \pm 2.28)$ & $15.2( \pm 2.29)$ & 0.689 \\
\hline$\beta$-PPA & $21(80.8 \%)$ & $35(87.5 \%)$ & $30(85.7 \%)$ & 0.751 \\
\hline Disc area $\left(\mathrm{mm}^{2}\right)$ & $1.41 \pm 0.21$ & $2.08 \pm 0.21$ & $2.75 \pm 0.24$ & $<0.0001$ \\
\hline Cup area $\left(\mathrm{mm}^{2}\right)$ & $0.36 \pm 0.25$ & $0.89 \pm 0.3$ & $1.42 \pm 0.35$ & $<0.0001$ \\
\hline Rim area $\left(\mathrm{mm}^{2}\right)$ & $1.05 \pm 0.22$ & $1.19 \pm 0.23$ & $1.33 \pm 0.26$ & 0.001 \\
\hline Cup/disc area ratio & $0.24 \pm 0.16$ & $0.42 \pm 0.12$ & $0.51 \pm 0.1$ & $<0.0001$ \\
\hline Cup volume $\left(\mathrm{mm}^{3}\right)$ & $0.07 \pm 0.07$ & $0.26 \pm 0.15$ & $0.44 \pm 0.22$ & $<0.0001$ \\
\hline Rim volume $\left(\mathrm{mm}^{3}\right)$ & $0.32 \pm 0.11$ & $0.31 \pm 0.13$ & $0.3 \pm 0.12$ & 0.676 \\
\hline Mean cup depth & $0.24 \pm 0.11$ & $0.33 \pm 0.09$ & $0.37 \pm 0.1$ & $<0.0001$ \\
\hline Horizontal cup/disc ratio & $0.48 \pm 0.25$ & $0.67 \pm 0.15$ & $0.74 \pm 0.13$ & $<0.0001$ \\
\hline Vertical cup/disc ratio & $0.39 \pm 0.24$ & $0.6 \pm 0.14$ & $0.67 \pm 0.1$ & $<0.0001$ \\
\hline
\end{tabular}

$C C T$ central corneal thickness, $D$ dioptres, IOP intraocular pressure

Optic disc parameters were obtained with confocal scanning laser tomography (HRT3; Heidelberg Engineering)

Among groups 1, 2 and 3: Kruskal-Wallis test. Bold font indicates significant $p$ values $(p<0.05)$

Table 2 Bruch's membrane opening parameters of each group according to disc size

\begin{tabular}{|c|c|c|c|c|c|c|c|}
\hline \multirow[t]{2}{*}{ Characteristics } & \multirow{2}{*}{$\begin{array}{l}\text { Group 1: small disc } \\
(n=26)\end{array}$} & \multirow{2}{*}{$\begin{array}{l}\text { Group 2: regular disc } \\
(n=40)\end{array}$} & \multirow{2}{*}{$\begin{array}{l}\text { Group 3: large disc } \\
(n=35)\end{array}$} & \multicolumn{4}{|c|}{$p$ values } \\
\hline & & & & 1 vs. 2 & 1 vs. 3 & 2 vs. 3 & Among 3 \\
\hline BMO area $\left(\mathrm{mm}^{2}\right)$ & $1.72 \pm 0.45$ & $2.29 \pm 0.31$ & $2.91 \pm 0.31$ & 0.001 & $<0.0001$ & 0.0001 & $<0.0001$ \\
\hline BMO-MRW G & $314.96 \pm 60.38$ & $259.03 \pm 40.04$ & $236.74 \pm 31.21$ & 0.001 & $<0.0001$ & 0.030 & $<0.0001$ \\
\hline BMO-MRW T & $236.19 \pm 50.00$ & $189.25 \pm 32.99$ & $176.97 \pm 30.54$ & & & & $<0.0001$ \\
\hline BMO-MRW TS & $310.92 \pm 70.88$ & $265.05 \pm 50.01$ & $240.31 \pm 37.24$ & & & & $<0.0001$ \\
\hline BMO-MRW NS & $372.23 \pm 85.57$ & $306.50 \pm 58.12$ & $271.03 \pm 38.56$ & & & & $<0.0001$ \\
\hline BMO-MRW N & $338.58 \pm 75.23$ & $268.08 \pm 55.37$ & $239.29 \pm 42.90$ & & & & $<0.0001$ \\
\hline BMO-MRW NI & $364.65 \pm 85.91$ & $312.35 \pm 57.42$ & $284.97 \pm 53.15$ & & & & $<0.0001$ \\
\hline BMO-MRW TI & $323.96 \pm 89.16$ & $284.88 \pm 54.40$ & $278.49 \pm 42.30$ & & & & 0.006 \\
\hline BMO-fovea angle ${ }^{\circ}$ & $-6.19 \pm 2.88$ & $-6.74 \pm 3.81$ & $-6.84 \pm 3.18$ & & & & 0.741 \\
\hline
\end{tabular}

$B M O$ Bruch membrane opening, BMO-MRW Bruch membrane opening minimum rim width, $G$ global, $T$ temporal, $T S$ superotemporal, $N S$ superonasal, $N$ nasal, $N I$ inferonasal, $T I$ inferotemporal

*Among groups 1, 2 and 3: Kruskal-Wallis test. Bold font indicates significant $p$ values $(p<0.05)$

${ }^{\dagger}$ Group 1 vs group 2 or group 1 vs group 3 or group 2 vs group 3: Kruskal-Wallis test with Donn's post hoc test. Bold font indicates significant $p$ values $(p<0.05)$

\section{Correlations between BMO-MRW and RNFL thickness from each scan circle}

\section{Inner circle}

In group 1, BMO-MRWs and RNFL thickness showed no significant correlations in any region, including global and six Garway-Heath sectors (Spearman's rho $<0.348$, all $p>$
0.017). In group 2, significant correlations between global BMO-MRWs and global RNFL thickness were noted (Spearman's rho $=0.572, p<0.001$ ). Among the six Garway-Heath sectors, superotemporal, superonasal, inferonasal and inferotemporal sectors showed significant correlations $(\mathrm{rho}=0.474-0.660$, all $p<0.017)$ whereas other sectors did not (all $p>0.017$ ). In group 3 , significant correlations were found between BMO-MRWs and RNFL 
Table 3 Retinal nerve fibre layer thickness from each scan circle in each group according to disc size

\begin{tabular}{|c|c|c|c|c|c|c|c|}
\hline \multirow{2}{*}{ Characteristics } & \multirow{2}{*}{$\begin{array}{l}\text { Group 1: small } \\
\text { disc }(n=26)\end{array}$} & \multirow{2}{*}{$\begin{array}{l}\text { Group } 2 \text { : } \\
\text { regular disc } \\
(n=40)\end{array}$} & \multirow{2}{*}{$\begin{array}{l}\text { Group 3: large } \\
\text { disc }(n=35)\end{array}$} & \multicolumn{4}{|c|}{$p$ values } \\
\hline & & & & 1 vs. 2 & 1 vs. 3 & 2 vs. 3 & Among 3 \\
\hline \multicolumn{8}{|l|}{ Inner circle } \\
\hline RNFL G $(\mu \mathrm{m})$ & $97.65 \pm 10.53$ & $100.13 \pm 9.62$ & $103.77 \pm 10.26$ & & & & 0.087 \\
\hline RNFL T & $82.88 \pm 16.55$ & $76.50 \pm 10.80$ & $77.97 \pm 9.45$ & & & & 0.353 \\
\hline RNFL TS & $141.42 \pm 21.81$ & $139.93 \pm 26.09$ & $140.06 \pm 21.15$ & & & & 0.587 \\
\hline RNFL NS & $116.04 \pm 27.46$ & $120.73 \pm 22.79$ & $120.06 \pm 24.14$ & & & & 0.860 \\
\hline RNFL N & $63.81 \pm 17.82$ & $74.75 \pm 16.04$ & $81.37 \pm 11.63$ & 0.101 & $<0.0001$ & 0.090 & $<0.001$ \\
\hline RNFL NI & $104.38 \pm 21.61$ & $109.18 \pm 19.22$ & $115.06 \pm 19.58$ & & & & 0.147 \\
\hline RNFL TI & $157.31 \pm 22.71$ & $154.10 \pm 17.29$ & $158.74 \pm 21.41$ & & & & 0.449 \\
\hline \multicolumn{8}{|l|}{ Middle circle } \\
\hline RNFL G $(\mu \mathrm{m})$ & $84.12 \pm 9.22$ & $85.43 \pm 7.89$ & $89.23 \pm 8.30$ & & & & 0.073 \\
\hline RNFL T & $73.35 \pm 14.54$ & $67.25 \pm 9.48$ & $69.23 \pm 8.00$ & & & & 0.147 \\
\hline RNFL TS & $128.35 \pm 20.82$ & $126.48 \pm 20.29$ & $127.29 \pm 19.14$ & & & & 0.488 \\
\hline RNFL NS & $92.31 \pm 22.6$ & $98.03 \pm 20.74$ & $97.29 \pm 17.24$ & & & & 0.721 \\
\hline RNFL N & $54.5 \pm 13.78$ & $61.85 \pm 12.84$ & $68.11 \pm 9.23$ & 0.202 & $<0.0001$ & 0.035 & $<0.001$ \\
\hline RNFL NI & $84.62 \pm 18.27$ & $87.85 \pm 16.87$ & $92.77 \pm 16.62$ & & & & 0.234 \\
\hline RNFL TI & $137.77 \pm 19.73$ & $135.35 \pm 14.10$ & $142.40 \pm 16.29$ & & & & 0.172 \\
\hline \multicolumn{8}{|l|}{ Outer circle } \\
\hline RNFL G $(\mu \mathrm{m})$ & $73.65 \pm 9.34$ & $74.38 \pm 6.90$ & $78.06 \pm 7.31$ & & & & 0.110 \\
\hline RNFL T & $64.46 \pm 15.34$ & $60.98 \pm 8.09$ & $62.94 \pm 7.17$ & & & & 0.309 \\
\hline RNFL TS & $115.54 \pm 19.18$ & $114.23 \pm 17.26$ & $116.09 \pm 18.22$ & & & & 0.608 \\
\hline RNFL NS & $76.65 \pm 16.64$ & $81.05 \pm 17.28$ & $81.31 \pm 14.52$ & & & & 0.752 \\
\hline RNFL N & $47.02 \pm 12.45$ & $52.70 \pm 12.08$ & $58.23 \pm 7.88$ & 0.316 & 0.001 & 0.048 & 0.001 \\
\hline RNFL NI & $69.54 \pm 15.77$ & $71.28 \pm 14.05$ & $76.83 \pm 14.12$ & & & & 0.087 \\
\hline RNFL TI & $118.73 \pm 20.9$ & $121.05 \pm 12.90$ & $126.86 \pm 15.59$ & & & & 0.185 \\
\hline
\end{tabular}

$R N F L$ retinal nerve fibre layer, $G$ global, $T$ temporal, $T S$ superotemporal, $N S$ superonasal $N$ nasal $N I$ inferonasal, $T I$ inferotemporal Inner scan circle diameter: $3.5 \mathrm{~mm}$, Middle scan circle diameter: $4.1 \mathrm{~mm}$, Outer scan circle diameter: $4.7 \mathrm{~mm}$

*Among groups 1, 2 and 3: Kruskal-Wallis test. Bold font indicates significant $p$ values $(p<0.05)$

${ }^{\dagger}$ Group 1 vs group 2 or group 1 vs group 3 or group 2 vs group 3: Kruskal-Wallis test with Donn’s post hoc test. Bold font indicates significant $p$ values $(p<0.05)$ thickness in global and all six sectors (rho $=0.375-0.662$, all $p<0.017$ ) (Table 4, Fig. 1d).

\section{Middle circle and outer circle}

Correlation tendencies between BMO-MRWs and RNFL thicknesses from middle and outer scan circles in each group were the same as those from the inner scan circle (Table 4).

\section{Discussion}

In the present study, we investigated BMO-MRW and RNFL thickness from three scan circles according to optic disc size. The effect of optic disc size on BMO-MRW and RNFL thickness as well as their correlations was well demonstrated. For the first time, we found that the correlation between BMO-MRW and RNFL thickness differed significantly according to optic disc size. It was the strongest in the large disc group, followed by that in the regular disc group. It was the weakest in the small disc group. To the best of our knowledge, this is the first study to show overall relation between BMO-MRW and RNFL thickness from three scan circles regarding all ranges of optic disc size (small, regular and large). BMO-MRW differed significantly according to optic disc size. However, RNFL thickness from all three scan circles did not show significant differences according to disc size. The correlation between BMO-MRW and peripapillary RNFL thickness showed the same tendency when RNFL thickness was measured from each of three scan circles (inner, middle and outer).

In our study, BMO-MRW was thinner in the large disc than that in the regular-sized disc. This finding is consistent with the study of Enders et al. [17]. on BMO-MRW in large 
Table 4 Correlations between BMO-MRW and RNFL thickness in each circle scan

\begin{tabular}{lcll}
\hline Characteristics & $\begin{array}{l}\text { Group 1: small disc } \\
(n=26)\end{array}$ & $\begin{array}{l}\text { Group 2: regular } \\
\text { disc }(n=40)\end{array}$ & $\begin{array}{l}\text { Group 3: large } \\
\text { disc }(n=35)\end{array}$ \\
\hline Inner circle & & & \\
RNFL G $(\mu \mathrm{m})$ & $0.147(p=0.473)$ & $\mathbf{0 . 5 7 2}(\boldsymbol{p}<\mathbf{0 . 0 0 1 )}$ & $\mathbf{0 . 6 5 6}(\boldsymbol{p}<\mathbf{0 . 0 0 1})$ \\
RNFL T & 0.348 & 0.138 & $\mathbf{0 . 5 0 3}$ \\
RNFL TS & 0.066 & $\mathbf{0 . 6 6 0}$ & $\mathbf{0 . 6 6 2}$ \\
RNFL NS & 0.171 & $\mathbf{0 . 4 9 7}$ & $\mathbf{0 . 3 7 5}$ \\
RNFL N & 0.174 & 0.198 & $\mathbf{0 . 3 8 7}$ \\
RNFL NI & 0.213 & $\mathbf{0 . 4 7 4}$ & $\mathbf{0 . 6 0 5}$ \\
RNFL TI & 0.304 & $\mathbf{0 . 5 2 0}$ & $\mathbf{0 . 5 9 0}$ \\
Middle circle & & & \\
RNFL G $(\mu \mathrm{m})$ & 0.126 & $\mathbf{0 . 5 5 0}$ & $\mathbf{0 . 6 1 2}$ \\
RNFL T & 0.298 & 0.142 & $\mathbf{0 . 5 3 2}$ \\
RNFL TS & -0.093 & $\mathbf{0 . 6 8 4}$ & $\mathbf{0 . 6 3 6}$ \\
RNFL NS & 0.143 & $\mathbf{0 . 4 7 1}$ & $\mathbf{0 . 3 7 6}$ \\
RNFL N & 0.219 & 0.172 & $\mathbf{0 . 3 4 6}$ \\
RNFL NI & 0.136 & $\mathbf{0 . 4 0 2}$ & $\mathbf{0 . 5 6 1}$ \\
RNFL TI & 0.319 & $\mathbf{0 . 5 2 3}$ & $\mathbf{0 . 4 1 9}$ \\
Outer circle & & & \\
RNFL G $(\mu \mathrm{m})$ & 0.111 & $\mathbf{0 . 5 1 7}$ & $\mathbf{0 . 5 7 4}$ \\
RNFL T & 0.213 & 0.166 & $\mathbf{0 . 4 8 4}$ \\
RNFL TS & -0.187 & $\mathbf{0 . 6 4 8}$ & $\mathbf{0 . 5 9 7}$ \\
RNFL NS & 0.174 & $\mathbf{0 . 5 0 1}$ & $\mathbf{0 . 3 7 6}$ \\
RNFL N & 0.226 & 0.092 & $\mathbf{0 . 2 9 2}$ \\
RNFL NI & 0.199 & $\mathbf{0 . 4 3 5}$ & $\mathbf{0 . 5 1 6}$ \\
RNFL TI & 0.376 & $\mathbf{0 . 5 4 4}$ & $\mathbf{0 . 4 1 5}$ \\
\hline Val & &
\end{tabular}

Values are Spearman's correlation coefficient rho ( $p$ value)

Bold font correlation coefficients indicate statistical significance $(p<$ 0.017 , owing to multiple comparison)

$B M O-M R W$ Bruch's membrane opening-minimum rim width, RNFL retinal nerve fibre layer, $G$ global, $T$ temporal, $T S$ superotemporal, $N S$ superonasal, $N$ nasal, $N I$ inferonasal, $T I$ inferotemporal

discs (disc area $>2.45 \mathrm{~mm}^{2}$ ). However, Enders et al. [17] did not directly compare BMO-MRW between large disc and regular-sized disc in the same study, although comparison was made with another study on normative eyes in white population by Chauhan et al. [12]. Another study by Enders et al. [18] has inspected BMO-MRW in microdiscs and reported that mean BMO-MRW in small discs (disc area $<1.63 \mathrm{~mm}^{2}$, mean BMO area: $1.45 \mathrm{~mm}^{2}$ ) in healthy subjects is $344.29 \mu \mathrm{m}$, concordant with our study (314.96 $\mu \mathrm{m}$ in group 1, disc area $<1.63 \mathrm{~mm}^{2}$, mean BMO area: 1.72 $\pm 0.45 \mathrm{~mm}^{2}$ ). A subsequent study by Enders et al. [19] also showed that macrodiscs (disc area $>2.43 \mathrm{~mm}^{2}$ ) had significantly smaller mean BMO-MRW than microdiscs (disc area $<1.63 \mathrm{~mm}^{2}$ ). They also described that BMO-MRW in normal eyes depended on disc size. In large optic discs, BMO-MRW was physiologically thinner [19]. However, they did not include regular-sized discs. They only compared small and large sized disc, unlike the present study. Our study directly investigated BMO-MRW using a full range of optic disc size, including small, regular, and large discs in a single ethnic group of East Asians.
In our study, no significant correlations were found between BMO-MRW and RNFL thickness in the small disc group in any region from any of the three scan circles. However, in the large disc group, significant correlations were shown in all sectors from all three scan circles. BMO is the outward border of the neuroretinal rim tissue at the optic disc through which RGC axons can pass [12]. It has been reported that BMO-MRW is advantageous to accurately reflect the amount of neural tissue from the optic nerve [20]. Although the amount of neuroretinal rim tissue is equivalent in discs of all sizes, disc area or BMO area is different by disc size. In small discs, BMO area and BMO circumference are smaller than those in regular discs. However, the same number of axons of RGC should pass through the BMO. As such, axons may be more crowded at BMO level. They might be more irregular in distribution. Actually, coefficient of variation of BMO area in small disc group was larger (0.26) compared with that in the regular $(0.13)$ or the large disc $(0.10)$ group in the present study. This larger variation in BMO area would translate into a larger variation of BMO-MRW in the small disc group than that in other groups. On the other hand, large discs have larger BMO area and larger circumference than regular or small discs. They might lead to thinner BMO-MRW and relatively regular distribution. It is currently unclear whether this effect of nerve fibre distribution can directly influence the correlation between BMO-MRW and RNFL in different disc size groups. Another explanation for this finding might be that nerve fibres in crowded discs cannot assume their "natural" diameter owing to lack of space. They might be compressed or forced to spare space. Nonetheless, these findings are possible because these BMO-based parameters can represent the true anatomy of the optic disc more correctly and therefore correspond more closely to the actual number of axons of RGC passing into the optic nerve than other parameters [21]. FoBMO angle was not significantly different among the three groups. Thus, it is not considered to have effect on the correlation. More studies are needed to inspect factors that affect the correlation between BMO-MRW values and RNFL thickness, especially in small discs.

These findings are clinically important in the diagnosis of glaucoma, especially in the early stage when structural tests do not always correspond with each other. For example, in a small disc, BMO-MRW is thicker than regular disc and its correlation with RNFL thickness may be weak. In some cases when BMO-MRW shows normal classification but RNFL thickness shows abnormal classification, this discrepancy can be partly owing to small optic disc size, which should be considered as one possible factor. Clinicians should be careful not to just consider these cases as normal or non-glaucomatous. Although BMO-MRW values are compared with normal values and already corrected for 
BMO size, there still exists significant difference in BMOMRW value according to optic disc size as shown in our study and many other previous studies [17-19, 22]. The correlation of colour classification of normal and abnormal between BMO-MRW and RNFL thickness did not always show consistency (data not shown owing to the limitation of pages).

However, it does not necessarily mean that BMO-MRW in small disc is of insignificant use. Enders et al. [19] have demonstrated that in microdiscs, there was a clear reduction of BMO-MRW with increasing VF defect at any given pathologic MD [19]. They also reported that BMOminimum rim area as another parameter did not show significant difference between large and small discs [19]. Moreover, we only investigated the correlation between BMO-MRW and RNFL in healthy non-glaucomatous eyes in the present study.

Tun et al. [22] have reported that OCT derived disc area has strong association with BMO-MRW by multivariate analysis. Mean disc area obtained by OCT was $1.93 \pm 0.37$ $\mathrm{mm}^{2}$ in their study [22], similar to BMO area of our regular disc group $\left(2.29 \pm 0.31 \mathrm{~mm}^{2}\right)$. However, they did not evaluate BMO-MRW according to different disc area group other than the normal range. In addition, their results were from an average population. Our study is unique in that it investigates BMO-MRW from the full range of disc area including small and large size which gives overall information about BMO-MRW according to optic disc size for clinicians.

RNFL thickness from each of inner, middle and outer scan circles did not show statistically significant differences among the three groups of disc size in the current study. In the study by Toshev et al. [20], sensitivity and specificity of global RNFL thickness from inner, middle and outer scan circles were similar (sensitivity, 78.18 81.82\%; specificity, 71.43 85.71\%). However, in their study, mean BMO disc area was $1.83 \pm 0.35 \mathrm{~mm}^{2}$ in the control group and $1.97 \pm$ $0.47 \mathrm{~mm}^{2}$ in the glaucoma group, similar to our small disc group $\left(1.72 \pm 0.45 \mathrm{~mm}^{2}\right)$ [20]. The present study has investigated RNFL thickness in all optic disc sizes of small, regular and large classified based on normative data range unlike the other previous studies.

The important strength of our study is that it provides overall perspective on the new parameter, BMO-MRW, in relation with RNFL thickness obtained from all three scan circles for the full range of disc size in the same ethnic population (East Asians). The present study not only included very small disc or very large disc, but also included regular size disc. This was different from other previous studies [12, 17-19, 22]. As references, these results are valuable to clinicians in diagnosing early glaucoma in patients with different disc sizes. It provides useful information for clinicians considering that four maps of OCT
(BMO-MRW, three scan circles of RNFL thickness) are now becoming available.

This study has several limitations mostly owing to its retrospective nature. First, we included only patients who had received a reliable visual field test. This might have influenced the accuracy of expected proportions. Another limitation was that it was a hospital-based study, not a population-based study, from a referral hospital of the province. Therefore, subjects included in this study might not represent all normal population. However, included subjects may represent relatively normal population in a clinical setting. The relatively small sample size of this study should also be taken into account, especially for the small disc group. This might be owing to the fact that it is more difficult to see patients with small discs in a glaucoma clinic because usually large discs and large cups are more often observed. Moreover, patients showing small discs often have localised RNFL defects. We excluded some patients with small discs owing to myopia of $<-6.0$ dioptres. We do not know the effect of excluding these patients.

In conclusion, we found that BMO-MRW differed significantly according to optic disc size. It was thicker in the small disc group and thinner in the large disc group than that in the regular disc group. Correlation between BMOMRW and RNFL thickness also showed different tendency according to disc size. The correlation was the strongest in the large disc group and modest in the regular-sized disc group. However, there were no significant correlations in the small disc group. There were no significant differences in RNFL thickness by disc size for each of the three scan circles except for nasal sectors. Thus, when we assess BMO-MRW in relation to RNFL thickness, disc size may need to be taken into consideration. A population-based study with a large number from multi-centre is needed in the future to draw more-definitive conclusions.

\section{Summary}

\section{What was known before}

- Bruch's membrane opening-minimum rim width (BMO$\mathrm{MRW}$ ), the new parameter, was introduced in the evaluation of the optic disc. However, its correlation with retinal nerve fibre layer (RNFL) thickness according to optic disc size has not been studied.

\section{What this study adds}

- Correlation between BMO-MRW values and RNFL thickness differed significantly according to optic disc size. It was the strongest in large disc, followed by regular disc, and none in small disc. The correlation was 
the same from each of the three RNFL scan circles of inner, middle and outer. RNFL thickness from each of the three scan circles showed no significant difference among disc size groups. When we assess the new parameter BMO-MRW in relation to RNFL thickness, disc size may need to be taken into consideration.

Acknowledgements This study received no financial support. H.C. and C.K. contributed to the design of this study. H.C., J.P. and C.K. conducted the study. H.C., J.P. and C.K. contributed to data collection, analysis, management and interpretation. H.C. and C.K. prepared the manuscript. This study was approved by the Institutional Review Board of Gyeongsang National University Changwon Hospital, Gyeongsang National University School of Medicine, Changwon, Republic of Korea. H.C. has full access to data obtained from this study and is responsible for the accuracy and integrity of data analysis. We also want to express our gratitude to Hyunhee Lee, a physician assistant, for assisting with data arrangement.

\section{Compliance with ethical standards}

Conflict of interest The authors declare that they have no conflict of interest.

Publisher's note: Springer Nature remains neutral with regard to jurisdictional claims in published maps and institutional affiliations.

\section{References}

1. Weinreb RN, Khaw PT. Primary open-angle glaucoma. Lancet 2004;363:1711-20.

2. AGIS Investigators. The Advanced Glaucoma Intervention Study (AGIS): 7. The relationship between control of intraocular pressure and visual field deterioration. Am J Ophthalmol 2000;130:429-40.

3. Kass MA, Heuer DK, Higginbotham EJ, Johnson CA, Keltner JL, Miller JP, et al. The Ocular Hypertension Treatment Study: a randomized trial determines that topical ocular hypotensive medication delays or prevents the onset of primary open-angle glaucoma. Arch Ophthalmol 2002;120:701-13.

4. Malik R, Swanson WH, Garway-Heath DF. 'Structure-function relationship' in glaucoma: past thinking and current concepts. Clin Exp Ophthalmol 2012;40:369-80.

5. Keltner JL, Johnson CA, Anderson DR, Levine RA, Fan J, Cello $\mathrm{KE}$, et al. The association between glaucomatous visual fields and optic nerve head features in the Ocular Hypertension Treatment Study. Ophthalmology 2006;113:1603-12.

6. Hood DC, Kardon RH. A framework for comparing structural and functional measures of glaucomatous damage. Prog Retin Eye Res 2007;26:688-710.

7. Chauhan BC, Burgoyne CF. From clinical examination of the optic disc to clinical assessment of the optic nerve head: a paradigm change. Am J Ophthalmol 2013;156:218-27 e2.

8. Chen TC. Spectral domain optical coherence tomography in glaucoma: qualitative and quantitative analysis of the optic nerve head and retinal nerve fiber layer (an AOS thesis). Trans Am Ophthalmol Soc 2009;107:254-81.
9. Povazay B, Hofer B, Hermann B, Unterhuber A, Morgan JE, Glittenberg $\mathrm{C}$, et al. Minimum distance mapping using threedimensional optical coherence tomography for glaucoma diagnosis. J Biomed Opt 2007;12:041204.

10. Reis AS, O'Leary N, Yang H, Sharpe GP, Nicolela MT, Burgoyne $\mathrm{CF}$, et al. Influence of clinically invisible, but optical coherence tomography detected, optic disc margin anatomy on neuroretinal rim evaluation. Invest Ophthalmol Vis Sci 2012;53:1852-60.

11. Strouthidis NG, Fortune B, Yang H, Sigal IA, Burgoyne CF. Longitudinal change detected by spectral domain optical coherence tomography in the optic nerve head and peripapillary retina in experimental glaucoma. Invest Ophthalmol Vis Sci 2011;52:1206-19.

12. Chauhan BC, Danthurebandara VM, Sharpe GP, Demirel S, Girkin CA, Mardin CY, et al. Bruch's membrane opening minimum rim width and retinal nerve fiber layer thickness in a normal white population: a multicenter study. Ophthalmology 2015;122:1786-94.

13. Chauhan BC, O'Leary N, Almobarak FA, Reis AS, Yang H, Sharpe GP, et al. Enhanced detection of open-angle glaucoma with an anatomically accurate optical coherence tomography-derived neuroretinal rim parameter. Ophthalmology 2013;120:535-43.

14. Mizumoto K, Gosho M, Zako M. Correlation between optic nerve head structural parameters and glaucomatous visual field indices. Clin Ophthalmol 2014;8:1203-8.

15. Pollet-Villard F, Chiquet C, Romanet JP, Noel C, Aptel F. Structure-function relationships with spectral-domain optical coherence tomography retinal nerve fiber layer and optic nerve head measurements. Invest Ophthalmol Vis Sci 2014;55:2953-62.

16. Gmeiner JM, Schrems WA, Mardin CY, Laemmer R, Kruse FE, Schrems-Hoesl LM. Comparison of Bruch's membrane opening minimum rim width and peripapillary retinal nerve fiber layer thickness in early glaucoma assessment. Invest Ophthalmol Vis Sci 2016;57:OCT575-84.

17. Enders P, Schaub F, Hermann MM, Cursiefen C, Heindl LM. Neuroretinal rim in non-glaucomatous large optic nerve heads: a comparison of confocal scanning laser tomography and spectral domain optical coherence tomography. $\mathrm{Br} \mathrm{J}$ Ophthalmol 2017;101:138-42.

18. Enders P, Schaub F, Adler W, Nikoluk R, Hermann MM, Heindl LM. The use of Bruch's membrane opening-based optical coherence tomography of the optic nerve head for glaucoma detection in microdiscs. Br J Ophthalmol 2017;101:530-5.

19. Enders P, Adler W, Schaub F, Hermann MM, Dietlein T, Cursiefen $\mathrm{C}$, et al. Novel Bruch's membrane opening minimum rim area equalizes disc size dependency and offers high diagnostic power for glaucoma. Invest Ophthalmol Vis Sci 2016;57:6596-603.

20. Toshev AP, Lamparter J, Pfeiffer N, Hoffmann EM. Bruch's membrane opening-minimum rim width assessment with spectraldomain optical coherence tomography performs better than confocal scanning laser ophthalmoscopy in discriminating early glaucoma patients from control subjects. J Glaucoma 2017;26:27-33.

21. Gardiner SK, Ren R, Yang H, Fortune B, Burgoyne CF, Demirel $\mathrm{S}$. A method to estimate the amount of neuroretinal rim tissue in glaucoma: comparison with current methods for measuring rim area. Am J Ophthalmol 2014;157:540-9 e1-2.

22. Tun TA, Sun CH, Baskaran M, Girard MJ, de Leon JM, Cheng $\mathrm{CY}$, et al. Determinants of optical coherence tomography-derived minimum neuroretinal rim width in a normal Chinese population. Invest Ophthalmol Vis Sci 2015;56:3337-44. 